\title{
An Efficient Mechanism Protocol for Wireless Sensor Networks by using Grids
}

\author{
Emad Ibbini, Student Member, Kweh Yeah Lun, Mohamed Othman, Zurina Mohd Hanapi, Nonmembers \\ University Putra Malaysia (UPM), Malaysia
}

\begin{abstract}
Multilevel short-distance clustering communication is an important scheme to reduce lost data packets over the path to the sink, particularly when nodes are deployed in a dense WSN (wireless sensor network). Our proposed protocol solves the problems of single hop paths in the TDTCGE (twodimensional technique based on center of gravity and energy) method, which addresses only single-hop problems and does not minimize distances between nodes by using multi-hop nodes with multilevel clustering grids to avoid dropped packets and to guarantee reliable paths without failures. In multilevel clustering grids, transmitted data are aggregated from lower-level grids to upper-level grids. In this paper, the proposed protocol obtains the optimal path for data transmission between cluster heads and the sink for heterogeneous WSNs. The cluster head nodes play an important role in forwarding data originating from other normal nodes that aggregate data to upper clusterheads. This routing approach is more efficient than other routing approaches, and it provides a reliable protocol for avoidance of data loss. In addition, the proposed protocol produces sleep and wakeup signals to the nodes and cluster heads via an MD (mediation device), thereby reducing energy consumption. Simulation results demonstrate the efficiency of the proposed method in terms of fewer dropped packets and high energy efficiency. The network environment overcomes the drawbacks of failure paths and provides reliable transmission to the sink.
\end{abstract}

Keywords-Multilevel; WSN; reliable; heterogeneous; routing

\section{INTRODUCTION}

Wireless sensor networks (WSNs) have many applications in fields such as agriculture, medical care and health care depending on the type of sensors installed. WSNs are crucial for gathering information necessary for smart devices that are part of pervasive computing, which is utilized in buildings, transportation and industrial systems. A pervasive sensor network consists of individual nodes (sensors) that can interact with the environment by sensing certain physical parameters. All sensor nodes generally have the same task. To complete their tasks, collaboration among nodes is required. Given that sink nodes can occasionally be outside the network, the data collected by sensors are transmitted to sink nodes that are part of the network. Sensors and sinks exchange packets through wireless communication.

Nodes cannot be connected easily to a wired power supply in many WSN applications; the nodes instead depend on onboard batteries [2]. In such cases, the energy efficiency of communication protocols is a crucial concern (i.e., figure of merit) because extended operation time is necessary. In other applications, power supply may not be a problem; consequently, other metrics (e.g., the accuracy of the delivered results) may be more relevant than energy efficiency.

A sensor is equipped with a radio transceiver or another wireless communication device that transmits and receives data over a wireless channel. A sensor also has a controller for manipulating data and memory for storing software and temporary data. A sensor commonly uses a battery as its energy source.

The concept of a WSN is based on a simple equation [3]: Sensing $+\mathrm{CPU}+$ Radio $=$ many applications. However, to create an effective WSN, the combination of sensors, radios, and CPUs requires in-depth understanding of the capabilities and limitations of hardware components and networks. WSNs face several problems that may not occur in other types of networks. Power constraints are a major concern. Communication is the most energy-intensive task a node performs. Nodes must compete for a share of the limited bandwidth available. Networking protocols attempt to reduce energy consumption by two means: neglecting certain communication tasks or turning off the radio transceiver when communications are unnecessary [1].

WSNs combine the latest advances in low-power microsensors and short-range wireless radios to yield an attractive new technology. WSNs enable a number of sensing and monitoring services in vital areas such as industrial production, home security +and in traffic and environmental monitoring. In addition, some of nodes be in sleep mode most of time to save energy as B-Mac [19].

The proposed protocol is an efficient Clustering Protocol for Heterogeneous energy nodes which divided into levels. There are many examples of Heterogeneous Oblivious Protocols, some of these examples Sep Protocol, ECHERP Protocol, Evolutionary Algorithms (EAs) and EECB (EnergyEfficient Chain-Based routing protocol) [13]-[18].

The rest of the manuscript provides some of the related works and describes the methodology of the proposed protocol then discusses the results with conclusions.

\section{LITERATURE REVIEW}

The proposed protocol is compared with the TDTCGE [7] protocol.

- Two-Dimensional Technique based on Center of Gravity and Energy (TDTCGE) [7].

This protocol uses two-dimensional techniques. The centers of gravity and energy for each grid are computed. The 
optimal node is selected to be the cluster head $(\mathrm{CH})$ because this node is the nearest to one of the centers. The TDTCGE protocol addresses the distance problem, particularly the distance of the $\mathrm{CH}$ from the BS. However, the problem of idle listening is overlooked. The results of this protocol indicate that both the lifetime and energy consumption are enhanced.

- In CRCWSN [8] this protocol uses two different techniques for selecting cluster head $(\mathrm{CH})$ that has been initially used by genetic algorithm and reclustering technique.

\section{A. Network Model}

For this study, we randomly deploy $\mathrm{N}$ sensor nodes in a monitored area and assume that the sensor network has the following characteristics:

1) The position of the BS in the sensor network is fixed.

2) All nodes are heterogeneous and stationary and have different initial supplies of energy.

3) All the nodes are randomly deployed in the target area, and each can establish a connection with the sink.

\section{B. Energy Consumption}

LEACH [4], [5] includes a first-order radio model that can be utilized for calculating hardware energy dissipation. For comparative purposes, this paper uses the same model. In this model, the energy consumptions of radios for sending and receiving data are both expressed as Eelect; the free space and the multi-path fading channel models with respective amplifying indexes $\varepsilon$ fs and $\varepsilon \mathrm{mp}$ are used; the energy consumption of data fusion is denoted by EDA [9]-[11]. The energy spent by a node that transmits an 1-bit packet over distance $d$ is calculated using the Heinzelman model. This model states that for each node to transmit L bits of data a distance d from itself, Et energy is consumed:

$$
\begin{gathered}
E t=L * \text { Eelect }+L * \in m p * d 4 d \geq d 0 . \\
\quad \text { else } \\
E t=L * \text { Eelect }+L * \in f s * d 2 \quad d<d 0 .
\end{gathered}
$$

The energy required to receive $L$ bits of data equals.

$$
E r=L * \text { Eelec. }
$$

The parameters are defined as follows:

$d 0$ : crossover distance

Eelect: energy necessary for activating electronic circuits $\in m p, \in f s$ : sensitivity and noise in the receiver, respectively.

\section{PROPOSED PROTOCOL}

In the proposed protocol, the target area is divided into grids, with each grid consisting of a cluster. Using grids reduce distance between nodes within cluster. Each cluster has a $\mathrm{CH}$ and connected member nodes. A mediation device (MD) node also exists which is intelligent device [6]; this node schedules and manages the nodes and $\mathrm{CH}$. After performing its task, the MD node synchronizes the nodes and the $\mathrm{CH}$. This node mostly keeps the $\mathrm{CH}$ and other nodes in sleep mode. The $\mathrm{CH}$ is awakened only for a short time to receive packets. The $\mathrm{CH}$ aggregates the data from the nodes and transmits them to the BS (see Fig. 5). Nine grids are established to ensure a high transmission data rate and to minimize the overall overhead.

The WSN environment is separated into 9 grids in the proposed protocol. Each grid consists of two dimensional centers (centers of gravity and energy). These two points are computed in each grid using the following two formulas (center of gravity and energy center) (see (1), (2), (3), (4)):

$$
X g c=(x 1 m 1+x 2 m 2) /(m 1+m 2)
$$

$$
\text { Ygc }(y 1 m 1+y 2 m 2) /(m 1+m 2) \text {. }
$$

If there are more than two object masses, then the formulas are represented as follows:

$$
\begin{gathered}
\text { Xgc }=\operatorname{sum}\left(\begin{array}{c}
X-\text { coordinate }(\text { node }) * \text { node } \\
- \text { mass }) / \text { all Mass. }
\end{array}\right. \\
Y g c=\operatorname{sum}(Y-\text { coordinate }(\text { node }) * \text { node } \\
\quad-\text { mass }) / \text { all Mass. }
\end{gathered}
$$

The center of energy for each grid is obtained by calculating the center of energy for the two (or more) points.

a) sum (X-coordinate(node) * node-mass) / node-count

b) sum (Y-coordinate(node) * node-mass) / node-count

The proposed protocol has the following rules:

$\mathrm{CH}$ : a super node that organizes all the nodes and aggregates data.

Centers of gravity and energy: center points used for reducing the distances between the nodes and $\mathrm{CH}$ to choose the optimal $\mathrm{CH}$. $\mathrm{CH}$

MD node: inelegant node that synchronizes the nodes and

By calculating the formulas for each center, the dimensional centers are included in each grid.

The center of gravity pertains to the average point of the object weight [17] (see Fig. 1).

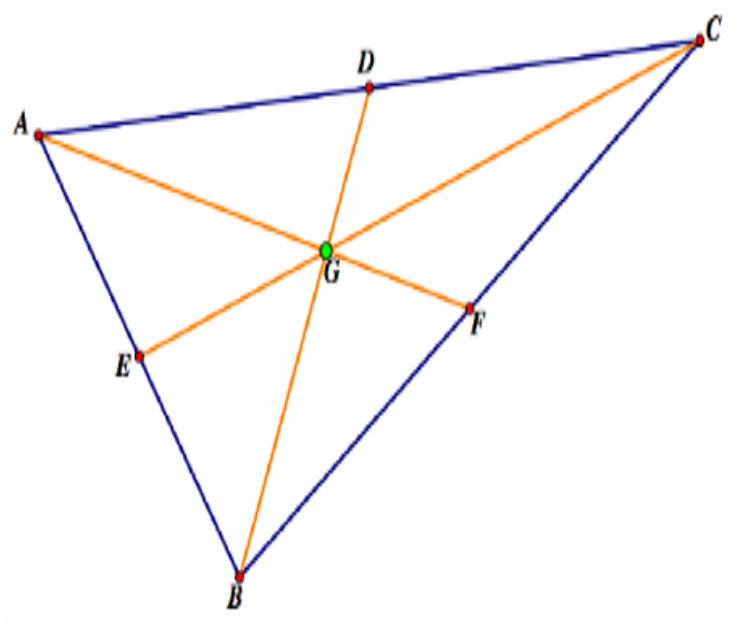

Fig. 1. Center of gravity. 
The proposed GMD protocol has two phases: setup and steady state. In the former, the network is divided into nine grids that result in four clusters. Each cluster has two centers (gravity and energy), one $\mathrm{CH}$, and undetermined nodes with different energies. An MD node is also present in each grid (see Fig. 2).

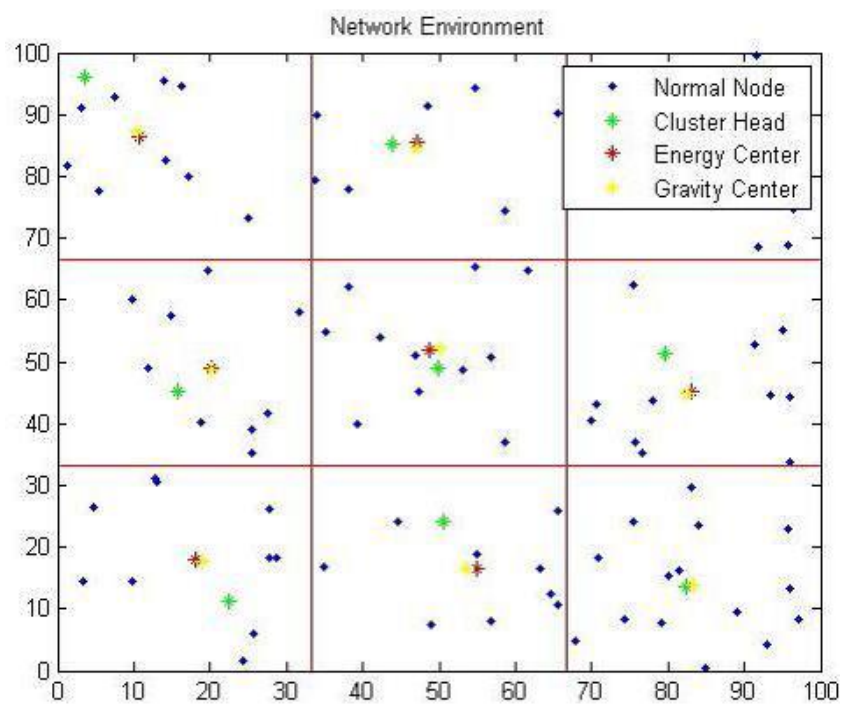

Fig. 2. Network environment.

\section{A. Setup Phase}

In the setup phase, the nodes are distributed randomly in the grids. After setup, the sink and centers are identified along with the $\mathrm{CH}$. The node closest to the sink and the energy center is selected as the $\mathrm{CH}$. The center of gravity should also be the closest to the BS for the node to be the $\mathrm{CH}$. If the node is far from the center of gravity but is the closest to the center of energy, it can be the $\mathrm{CH}$. If this center is also the nearest to the BS, then the node that has the most energy is the $\mathrm{CH}$. The proposed Protocol has three types of $\mathrm{CHs}$ in each level: normal, advanced, and super CH's. The ranking of CHs is according to the distance of the nodes from the BS and how close the heterogeneous nodes are to the energy center; the node that has the most energy is the $\mathrm{CH}$. The weights of normal $\mathrm{CHs}$ are accordingly less than the weights of advanced $\mathrm{CHs}$ and super CHs.

\section{B. Steady-State Phase}

In the steady-state phase, the BS broadcasts the address and ID number of each node. An MD node that works as a node mediator is present in each grid. This node is responsible for scheduling, managing the suggested routing protocol, and treating the synchronization operations between the nodes and the $\mathrm{CH}$. The MD node performs its operations in the grids in two cases: when the nodes have no data and when they have data.

Case 1: If nodes in each grid have no data to send to the $\mathrm{CH}$, then the MD node keeps the nodes and the $\mathrm{CH}$ in sleep mode most of the time by transmitting a sleep signal (see Fig. 3).

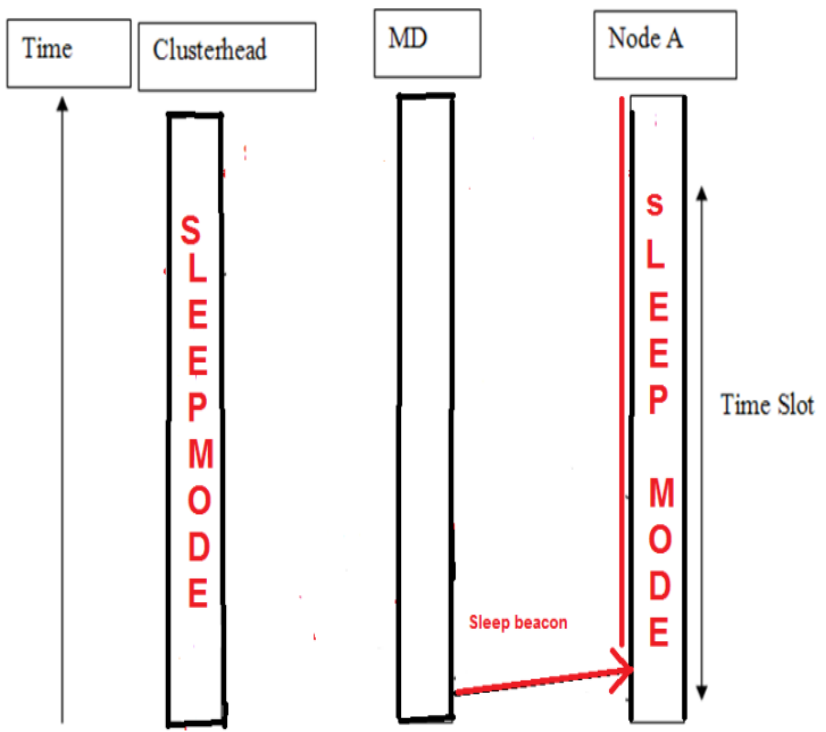

Fig. 3. Case 1: No Data.

Case 2: If new data must be received, the MD node sends a wake-up signal to the nearest node. The wake-up signal is also sent to the $\mathrm{CH}$. The $\mathrm{CH}$ and the nodes are in sleep mode most of the time. To receive data, they wake up for a short time. The MD node produces a binary digit "0" for sleep to nodes which doesn't have data and " 1 " for wake up to nodes have data notify that MD node is intelligent device. The nodes then wait for their time slots to transmit data on their time-division multiple accesses (TDMA) [12] schedule. In this schedule, the nodes that have data when the binary digit is " 1 " are prioritized. Accordingly, the MD node transmits a wake-up signal to the nearest node up to the farthest one to minimize the delay in sending data and simultaneously save energy (see Fig. 4).

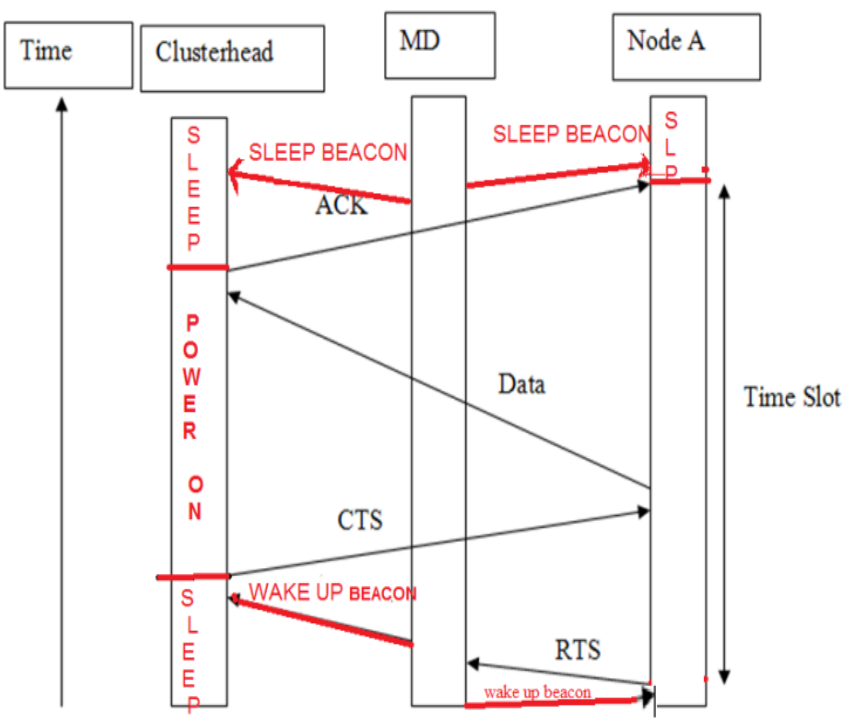

Fig. 4. Case 2: With Data.

The MD node creates a wake-up signal containing the address of the node that must transmit a packet. The signal is passed to the $\mathrm{CH}$, which in turn responds by transmitting a 
clear-to-send (CTS) signal to the source node. The source node then transmits the data directly to the $\mathrm{CH}$. After receiving the data, the $\mathrm{CH}$ sends an acknowledgment back to the source node $\mathrm{A}$, thus signaling that the transmission is completed. According to Fig. 3, in this proposed study there is multilevel clusterheads and Multilevel MD (see Fig. 5).

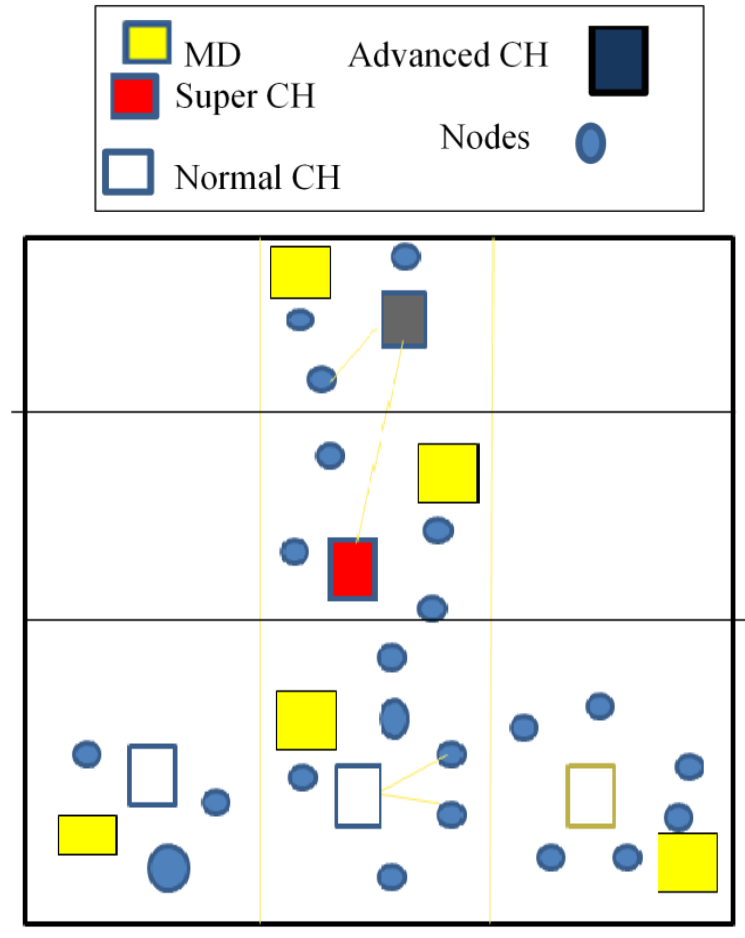

Fig. 5. Multilevel clusterhead grids.

In the first level, the normal $\mathrm{CH}$ aggregates the transmission data from normal nodes; this occurs after the MD node lets the nodes sleep most of time unless they have data. When the nodes have data, the MD will wake them up and refer them to the normal $\mathrm{CH}$. All normal $\mathrm{CHs}$ will forward the data to the advanced $\mathrm{CH}$; this also aggregates data from its cluster nodes. The MD node then lets normal $\mathrm{CHs}$ return to sleep mode. The advanced $\mathrm{CH}$ will then forward the aggregated data to the super $\mathrm{CH}$, which will transmit them to the BS. To overcome the above problem, an efficient multihop heterogeneity protocol is proposed to obtain an optimal path with no failures or dropped packets between the CHs and the BS during data transmission. This will reduce the transmission path instead of transmitting directly from a normal $\mathrm{CHs}$ to the $\mathrm{BS}$, as in the TDTCGE protocol. The second level advances cluster head nodes, which are allowed to communicate with the third level super cluster head node in its TDMA time slot; the same is followed for level 1 nodes to level 2 CHs. In level 2, the advanced $\mathrm{CH}$ node performs data aggregation to remove replicate data. In level 2 node sends, the aggregated data advance to the level $3 \mathrm{CH}$ in its TDMA time slot. In level 3 super $\mathrm{CH}$ nodes, the data use network coding; they are forwarded to the sink. Owing to a multi-hop link [20] between level $3 \mathrm{CHs,} \mathrm{data} \mathrm{forwarded} \mathrm{to} \mathrm{the} \mathrm{sink}$ increase the network traffic. The network environment overcomes the disadvantages and provides reliable transmission to the sink.

\section{SETUP PHASE}

Algorithm:

Divide the network into two grids.

Find the center of gravity for each grid.

1. If node-count $=1$

a. The node that is nearer to BS participates in the grid computation.

2. If node-count $>1$

a. allMass $=$ calculate sum of the masses of all nodes in grid

b.

b. sum (X-coordinate(node) * node-mass) / allMass

c. sum (Y-coordinate(node) * node-mass) / allMass

3 . Find the center of energy for each grid.

1. If node-count $=1$

a. The node that is nearer to BS participates in grid computation.

2. If node-count $>1$

a. sum (X-coordinate(node) $*$ node-mass) / node-count

b. sum (Y-coordinate(node) * node-mass) / node-count

4. If Distance (normal nodes) $<=$ distance(center of energy, BS),

A. select most energetic node as Normal $\mathrm{CH}$

5. else If (Advanced nodes $<$ Advanced $\mathrm{CH}$ )

A. Select Advanced node as Advanced CH.

Else

B. Select Advanced node as Super $\mathrm{CH}$

\section{STEADY PHASE:}

\section{Algorithm:}

Steady State Phase: Algorithm

Repeat 1 . If the node is normal

MD broadcasts IP address and ID number to all nodes

If node $=$ data

MD produces wake-up signal

If Distance(node $<=\mathrm{CH}$ )

Node sends RTS to $\mathrm{CH}$

$\mathrm{CH}$ sends CTS to node

Node sends data to $\mathrm{CH}$

$\mathrm{CH}$ sends acknowledgment to nodes

Flag=1

a. node-energy $=$ node-energy - consumed energy when sending a message

2. Otherwise

a. node-energy = node-energy - aggregated energy consumed energy )when sending a message until no node has energy(

B. $($ Advanced-CH $)=$ Node-energy + Normal $\mathrm{CH}$-energy

C. $($ Super $-\mathrm{CH})=($ Advanced $-\mathrm{CH})+($ Advanced-Nodes-energy $)$ 


\section{PERFORMANCE MEASUREMENT}

The performance of the proposed protocol can be evaluated with a number of metrics.

\section{A. Performance Evaluation}

- Energy Consumption: The total numbers of energy consumed for packets transmitted and packet received during the rounds.

- Throughput: It measures the total data rate sent over the network, including the data rate sent from $\mathrm{CHs}$ to the sink node and that sent from the nodes to their CHs.

- Packet loss: Many causes of data loss would be bit errors in an erroneous wireless network or collisions due to network congestion when the channel becomes overloaded or large distance path to base station.

\section{B. Results and Discussion}

TABLE I. SIMULATION PARAMETER

\begin{tabular}{|ll|}
\hline Parameter & Value \\
\hline Network size & $100 * 100 \mathrm{~m}$ \\
\hline Ee & $50 \mathrm{~nJ} / \mathrm{bit}$ \\
\hline Tevent_all & $($ randi $(9,1, \mathrm{~m})+1) * 1 * 10^{-3} \mathrm{~m}$ \\
\hline T & 10 \\
\hline Pactive & $6 * 10^{-3} \mathrm{mw}$ \\
\hline Tdown & $1 * 10^{-3} \mathrm{~m}$ \\
\hline Psleep & $1 * 10^{-3} \mathrm{mw}$ \\
\hline L & $1000 \mathrm{bit}$ \\
\hline Do & $87 \mathrm{~m}$ \\
\hline Grids Number & 4 \\
\hline Mp & $0.0013 * 10^{-9}$ \\
\hline Fs & $10^{*-9} 10^{-9}$ \\
\hline Position of BS & $(75,125)$ \\
\hline Number of nodes & 100 \\
\hline
\end{tabular}

We use these parameters from Table I in matlab simulation to evaluate the MD Multilevel Proposed Protocol with MD one level and TDTCGE Protocol.

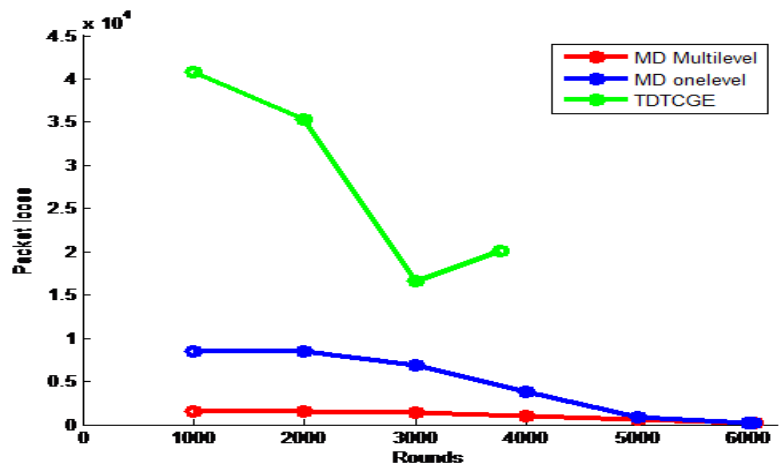

Fig. 6. Drop packets during rounds.

From Fig. 6, we can observe that the Proposed MD multilevel protocol is more reliable than MD onelevel protocol and TDTCGE which is the least of dropping packets between these two existing protocols. The percentage of errors with dropping packets during rounds for each protocol is $35 \%$ to MD Multilevel, $45 \%$ to MD one level and $65 \%$ to TDTCGE. The MD multilevel obtain the optimal path for data transmission to reach the base station to avoid loose date and guarantee reliable paths without failures.

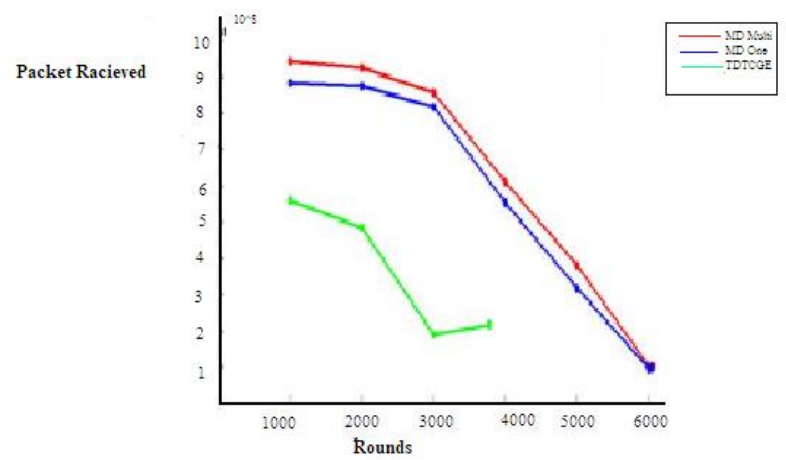

Fig. 7. Number of messages received during rounds.

From Fig. 7, we can observe that the Proposed MD multilevel protocol is more efficient than MD onelevel protocol and TDTCGE. The large volume of successfully messages received to $\mathrm{BS}$ by $\mathrm{MD}$ multilevel protocol more than these two existing protocols. The received successfully messages by MD-multilevel is $9.5 * 104$, MD-onelevel is $8.7 * 104$. The MD multilevel obtains the optimal path for data transmission to reach the base station.
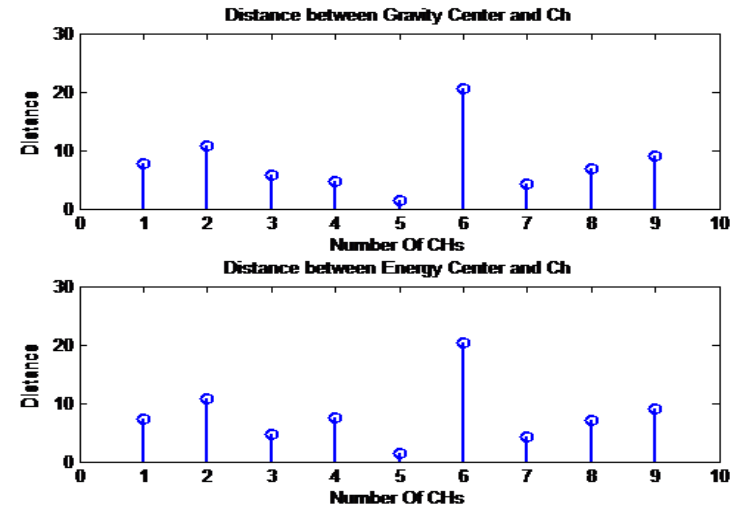

Fig. 8. Distance for MD (multi)
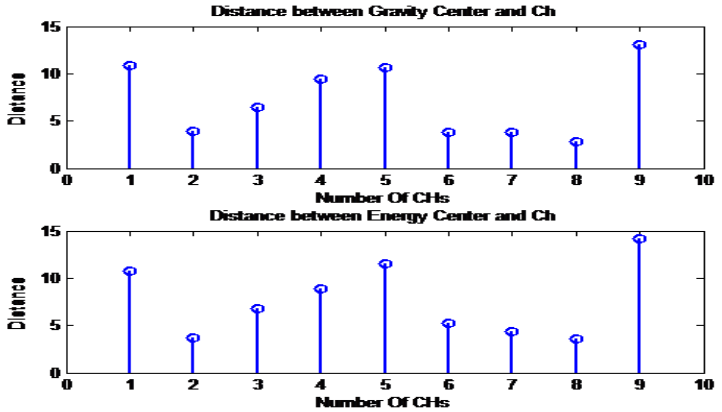

Fig. 9. Distance for MD (one level)

We observe from Fig. 8 and 9 above that for the MD multilevel protocol, all the clusterhead candidates were near 
the energy center points except for one candidate, CH6. However, in the MD-one level protocol, all clusterhead candidates were near except three: $\mathrm{CH} 1, \mathrm{CH} 5$ and $\mathrm{CH}$ 9. Thus, the MD-multilevel protocol was more precise in choosing an efficient clusterhead.

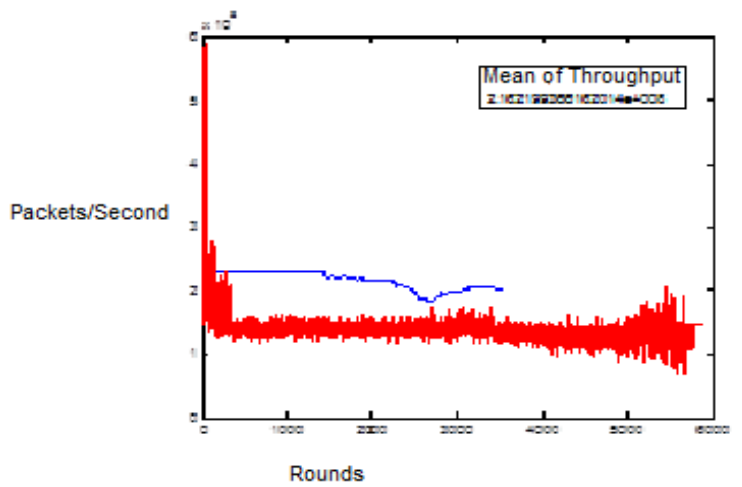

Fig. 10. Mean of throughput.

Fig. 10 compares the throughput values of the MD-one level protocol and the proposed MD multilevel protocol using nine grids for both. A long transmission time implies a low throughput. A large throughput represents a large number of messages delivered per unit time, regardless of whether the delivery was successful, i.e., Throughput $=($ Size of the packet / Transmission time). The TDTCGE protocol requires a long time to send data to the $\mathrm{BS}$, and it uses nine grids that result in a small throughput. This is due to the small number of nodes in each grid. The time required to reach the $\mathrm{BS}$ is thus reduced. The number of data packets received by the BS per unit time in the proposed MD protocol is greater than that received by the BS per unit time in the MD-one level protocol. The proposed protocol exhibits a higher mean throughput than the TDTCGE protocol. The mean throughput of the latter is $2.1621 * 108$, whereas that of the former is $2.5^{*} 10^{8}$ (see Fig. 11).

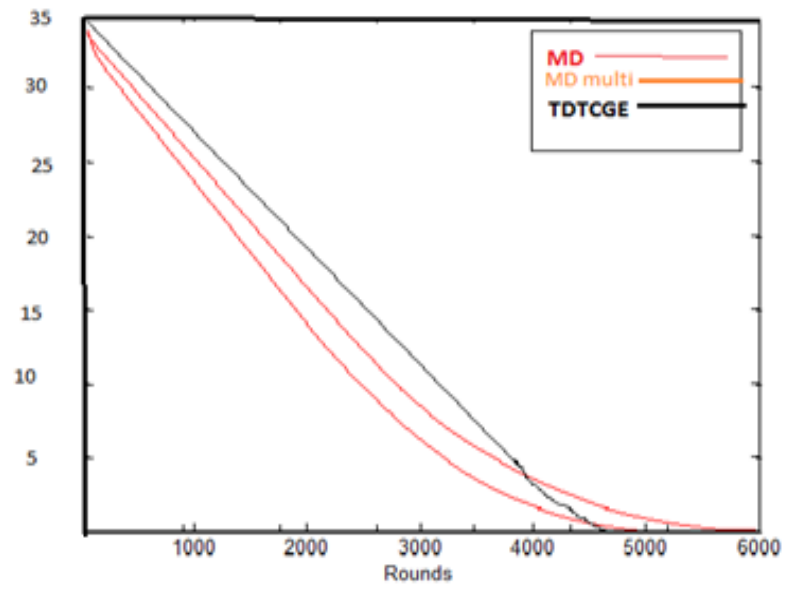

Fig. 11. Energy consumption.

Fig. 11 reveals that the proposed MD-multilevel protocol conserves more energy than the TDTCGE protocol. The former consumes 34 Joules in 5900 rounds, whereas the latter consumes 35 Joules in 4880 rounds. However, the MD-one level protocol saves more energy than the MD-multilevel and TDTCGE protocols; it consumes 33 Joules in 6050 rounds.

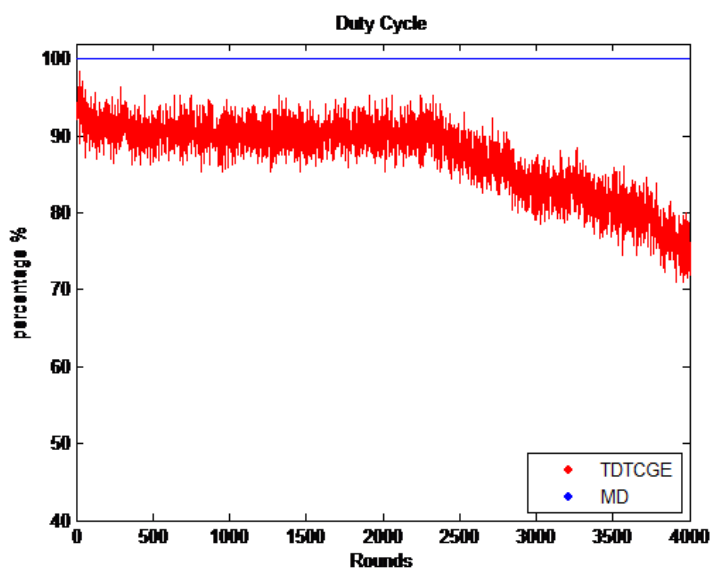

Fig. 12. Duty cycle.

The duty cycle in Fig. 12 pertains to the ratio of time, a node is in the active mode to the entire operational time. WSNs are typically engineered with low duty cycles to guarantee long node and network lifetimes. Therefore, most of the time, the nodes are in extended sleep modes with their radios turned off. Duty cycling limits the number of nodes that synchronously overhear a packet. Thus, the spatial reuse in the forwarding process is restricted. For the proposed and TDTCGE protocols, the resulting duty cycle for the given nodes was $80 \%-90 \%$ during all rounds (see Fig. 12). The average duty cycle for all nodes was $85 \%$. The average duty cycle was $100 \%$ and $95 \%$ for the first 400 rounds and for rounds 401-2300, respectively. This percentage then decreased from $95 \%$ to $75 \%$ at 4000 rounds.

\section{CONCLUSIONS}

The proposed protocol is more reliable than existing protocols for multilevel heterogeneous WSNs. Comparing TDTCGE, MD-one level and MD-multilevel protocols, the proposed protocol was evaluated in terms of the messages received by the sink and the network lifetime. The proposed MD-multilevel protocol is well organized in establishing multi-hop communication within grids using link path correlation along with the TDMA time slot. Moreover, multihop communication between cluster heads is well controlled by the MD node. Because heterogeneous nodes are usually chosen as clusterhead candidates, incorporating energyconsuming tasks on those nodes increases the number of messages received by the network sink. The simulation results illustrate the efficiency of the multilevel MD protocol compared with the existing protocols in terms of reducing both the energy consumption and the number of dropped packets and hence guarantee the reliability of the proposed multilevel protocol to deliver data without failure.

\section{FUTURE WORK}

In the future Research Plans that how to mix fuzzy logic system with MD node and adding grids and Centres. We highlight some interesting on the future research directions: 
- Currently, the proposed protocols designed mainly to solve this problem as idle listening and delay with throughput by adding MD node alone also adding Mathematical model will improve the lifetime of the network $90 \%$ than TDTCGE.

- In addition, in the Proposed Schemas we will add fuzzy logic with three criteria's to the grids and Centers that the proposed schema minimize the distance and saving more energy which give us a better energy efficiency than TDTCGE Protocol.

- Furthermore, when we plan to mix Fuzzy logic with MD node and adding mathematical model to the grids and Centres which that solve all the problems in this research in addition, solving the problems of reliability and collisions.

\section{REFERENCES}

[1] David Culler, Deborah Estrin, "Overview Of Sensor Network", Published By IEEE International Conference On Robotics And Automation, 5, 201-290,2004.

[2] Holger Karl, Andreas Willing, "Protocols And Architecture For Wireless Sensor Network", John Wiley \& Sons, 2005.

[3] Jason Lester Hill, "System Architecture For Wireless Sensor Networks", University Of California, Berkeley, 2003.

[4] Wei Ye, John Heidmann, Deborah Estrin, "An Energy-Effficient MAC Protocol For Wireless Sensor Network", Information Science Institue (ISI), University Of Southern California. In Proceedings Of The IEEE Infocom, 1567-1576, 2002.

[5] Wendi Heinzelman, AnanthaChandrkasan, Hari Balakrishnan, "Energy-Efficient Communication Protocol For Wireless Microsensor Network", Proceedings Of The 33rd Annual Hawaii International Conference On System Sciences, 2000.

[6] Emad Mohammed Ibbini, "An Efficient Mechanism For Saving Energy Of LEACH Protocol In WSN", Masters Thesis, Computer Science, Jordan University Of Science \& Technology, Jordan, 2010.

[7] AtefehHeydariyan, Amir Abbas Baradaran, ElhamRezaei, 2013," TDTCGE: Two Dimensional Technique Based On Center Of Gravity And Energy Center In Wireless Sensor Network", Journal Of Basic And Applied Scientific Research ( ISSN: 2090-4304) Pages:194201,2013.

[8] Delavar, A.G. And A.A. Baradaran, "CRCWSN: Presenting A Routing Algorithm By Using Re-Clustering To Reduce Energy Consumption" , In WSN. International Journal Of Computers Communications \& Control, 2013.(ISI)(ISSN 1841-9836) 8(1): P. 6169. , 102-114

[9] I. F. Akyildiz,W. Su, Y. Sankarasubramaniam, and E. Cayirci, "A Survey on Sensor Netowrks," IEEE Communications Magazine, 40, 8, 2002.

[10] M. BaniYassein And H. Mistareehi, "Improvement On The Lifetime Of The WSN Using Energy Efficiency Saving Of Leach Protocol (New Improved LEACH)", Sensors \& Transducers Journal, 130(7): 142-154. , 2011.

[11] Emad Mohammed Ibbini, Kweh Yeah Lun, Mohamed Othman, Zurinah Mohd Hanapi, Mohammed S Ibbini "A Survey Of Routing MAC Techniques For Wireless Sensor Networks Routing Protocol" Journal Of Theoretical And Applied Information Technology (JATIT), 2015.
[12] A 2 RATHNA. R 1 AND SIVASUBRAMANIAN. "IMPROVING ENERGY EFFICIENCY IN WIRELESS SENSOR NETWORKS THROUGH SCHEDULING AND ROUTING", International Journal Of Advanced Smart Sensor Network Systems ( IJASSN), vol 2, No.1, January 2012.

[13] G. Smaragdakis, I. Matta, A. Bestavros, "SEP, a stable election protocol for clustered heterogeneous wireless sensor networks", in: Second International Workshop on Sensor and Actor Network Protocols and Applications (SANPA 2004), Boston, MA, 2004.

[14] Enan A. Khalil, Bara'a A. Attea ,"Energy-aware evolutionary routing protocol for dynamic clustering of wireless sensor networks. Swarm and Evolutionary Computation", 1(4): p. 195-203,2011.

[15] Stefanos A. Nikolidakis , DionisisKandris, Dimitrios Vergados and Christos Douligeris, "Energy Efficient Routing in Wireless Sensor Networks Through Balanced Clustering". Algorithms, 6, 29-42; doi:10.3390/a6010029, 2013.

[16] Aimin Wang, Dailiang Yang, Dayang Sun :"A clustering algorithm based on energy information and cluster heads expectation for wireless sensor networks". Computers and Electrical Engineering 38, 662-671, 2012.

[17] Bara'a, A. Attea, and Enan A. Khalil. "A new evolutionary based routing protocol for clustered heterogeneous wireless sensor networks." Applied Soft Computing 12.7, 1950-1957, ,2012:

[18] Sheikhpour, Razieh, and Sam Jabbehdari. "An energy efficient chainbased routing protocol for wireless sensor networks." KSII Transactions on Internet and Information Systems (TIIS) 7.6, ): 1357-1378, 2013.

[19] Cano, Cristina, et al. "A low power listening MAC with scheduled wake up after transmissions for WSNs." Communications Letters, IEEE 13.4, 221-223, 2009.

[20] Zhang, Xu, et al. "An efficient hop count routing protocol for wireless ad hoc networks." International Journal of automation and computing 11.1,93-99,2015.

\section{AUTHOR'S PROFILE}

EMAD Mohammed Ibbini is a Ph.D Student in Computer Network (UPM), M.SC in Computer Science (JUST) Bachelor in Computer of Information Systems in (JUST), Specialized in WSN and ad-hoc Networks.

Dr. Kweh Yeah Lun, Dr. B.Sc. in Comp. Sc. (UPM), M.Sc. (UPM), Ph.D (UPM) (in Distributed Computing, Parallel and Distributed Algorithm), Tel: 03-89471797

Prof. Mohamed Othman is an Associate Professor of Computer Science in the Dept. of Communication Technology and Network, Universiti Putra Malaysia (UPM), since 2001. In April 2008, he was appointed as a deputy director of InfoComm Development Centre (iDEC) at the same university. He received his Ph.D in Computer Science from the Department of Industrial Computing, Universiti Kebangsaan Malaysia with distinction (Best PhD Thesis in 2000 awarded by Sime Darby Malaysia and Malaysian Mathematical Science Society) in 1999. During his PhD study, he has contributed and published twelve journal papers in which five were in impact factor journals and the others were in cited and indexed journals. Since 1989, in total, he had published 110 journal papers and more than 300 conference papers, both at national and international levels. He has expertise in several areas of computer science such as parallel and distributed algorithms, grid computing, highspeed computer network and scientific computing.

Associate Prof. Dr Zurina Mohd Hanappi, B.Comp. Sc. (Strathclyde), M.Sc. (UPM), Ph.D (UKM, Specialized, Computer System Engineering, Network Security, Distributed Computing). 